\title{
Pingüinos fósiles (Pygoscelis calderensissp. nov.) en la Formación Bahía Inglesa (Mioceno Medio-Plioceno), Chile
}

\section{Carolina Acosta Hospitaleche}

Martín Chávez

Omar Fritis
División Paleontología Vertebrados, Museo de La Plata, Paseo del Bosque s/n, 1900, La Plata, Argentina-CONICET acostacaro@museo.fcnym.unlp.edu.ar

Instituto de Zoología, Universidad Austral de Chile, Av. México 9662, La Florida, Santiago, Chile paleoaeolos@gmail.com

Facultad de Ciencias Naturales y Oceanográficas, Universidad de Concepción, Chile ofritis@yahoo.com

\section{RESUMEN}

Se describen tres nuevos cráneos de pingüinos (Spheniscidae), asignados a Pygoscelis calderensis sp. nov., procedentes de la Formación Bahía Inglesa del Mioceno Medio-Plioceno, ubicada al sur de la localidad de Caldera, en la costa de la III Región de Atacama, Chile $\left(27^{\circ} 00^{\prime} \mathrm{S}, 70^{\circ} 45^{\prime} \mathrm{W}\right.$ a $\left.28^{\circ} 00^{\prime}, 71^{\circ} 00^{\prime} \mathrm{W}\right)$. Este hallazgo permite ampliar la distribución cronológica y geográfica del género, previamente conocido desde el Plioceno Tardío de Nueva Zelanda, y constituye la mención más septentrional del mismo. Este registro, sumado a otras evidencias faunísticas, indica la existencia de períodos o corrientes oceánicas frías durante el Neógeno.

Palabras claves: Paleombiente, Spheniscidae, Pygoscelis, Mioceno Medio-Plioceno, Bahía Inglesa, Chile.

\section{ABSTRACT}

Fossil penguins (Pygoscelis calderensis sp. nov.) in the Bahía Inglesa Formation (Middle MiocenePliocene), Chile. Three new penguin skulls (Spheniscidae), assigned to the new species Pygoscelis calderensis sp. nov. from the Bahía Inglesa Formation of Middle Miocene-Pliocene age located south of Caldera on the coast of the III Región de Atacama, Chile $\left(27^{\circ} 00^{\prime} \mathrm{S}, 7^{\circ} 45^{\prime} \mathrm{W}\right.$ to $\left.28^{\circ} 00^{\prime}, 71^{\circ} 00^{\prime} \mathrm{W}\right)$, are described. This finding broadens the geographic and chronologic distribution of the genus, constituting its most northern record. Before the present work, the genus was known from the Late Pliocene of New Zealand. This record, together with other faunistic evidences, suggests the existence of periods or cold oceanic currents during the Neogene. 


\section{INTRODUCCIÓN}

El orden Sphenisciformes (pingüinos), que comprende una única familia (Spheniscidae), constituye un grupo de aves con caracteres altamente derivados. La totalidad de las especies conocidas son aves no voladoras y con particulares adaptaciones al buceo submarino (Martínez, 1992). Este orden está restringido al Hemisferio Sur y se encuentra asociado a corrientes oceánicas frías, gracias a las cuales extienden su rango de distribución desde la Antártica hasta las islas Galápagos en el Ecuador. Los registros más tempranos del orden aparecen en el Paleoceno Superior de la isla Seymour, Antártica (Tambussi et al., 2002); incrementando su frecuencia a partir del Eoceno en Sudáfrica, Australia, Antártica, América del Sur y Nueva Zelanda, siendo estas dos últimas áreas las de mayor riqueza en términos de diversidad y abundancia (Simpson, 1975; Acosta Hospitaleche, 2004).

Los primeros estudios paleontológicos de Sphenisciformes, sugirieron la existencia de al menos 4 subfamilias extintas 'Palaeeudyptinae' y 'Anthropornithinae' registradas durante el Eoceno Tardío-Oligoceno, 'Paraptenodytinae' cuyos registros se extienden entre el Eoceno Tardío y el Plioceno Temprano y 'Palaeospheniscinae' cuyo biocrón va del Mioceno Temprano al Plioceno Medio (Simpson, 1946; Acosta Hospitaleche, 2004; Acosta Hospitaleche y Canto, 2005), más una quinta subfamilia, 'Spheniscinae', compuesta por los géneros modernos que presentan sus primeros registros en el Mioceno Medio (Simpson, 1946; Acosta Hospitaleche, 2004; Acosta Hospitaleche y Canto, 2005; Stucchi, 2002; Stucchi et al., 2003).

Esta clasificación a nivel de subfamilia no ha sido consensuada por la totalidad de los investigadores por creerse que se trataría de agrupamientos artificiales (Simpson, 1975; Olson, 1986; Fordyce y Jones, 1990; Fordyce, 1991). Sin embargo, estos agrupamientos reúnen a las especies fósiles y actuales de una manera clásica, que resulta útil a fines descriptivos (Myrcha et al., 1990; Acosta Hospitaleche, 2004). Resulta oportuno aclarar que el empleo de estas categorías no implica necesariamente algún tipo de relación filogenética.

Los estudios realizados en la costa atlántica de la Patagonia Argentina han determinado la pre- sencia de varias especies atribuibles a los 'Palaeospheniscinae' y 'Paratenodytinae' en la Formación San Julián del Eoceno Tardío-Oligoceno Temprano (Acosta Hospitaleche, 2005), la Formaciones Chenque, Gaiman y Monte León del Mioceno Temprano (Acosta Hospitaleche, 2004; Acosta Hospitaleche et al., 2004) y la Formación Puerto Madryn del Mioceno Tardío temprano (Acosta Hospitaleche, 2003; Cione y Tonni, 1981), mientras que en la costa pacífica sólo recientemente se ha documentado la presencia de especies asignadas a los 'Spheniscinae', 'Palaeospheniscinae' y 'Paraptenodytinae' en las Formación Bahía Inglesa de Chile (Acosta Hospitaleche et al., 2002; Walsh, 2004; Acosta Hospitaleche y Canto, 2005; Chávez, 2005a), la Formación Pisco del Mioceno Tardío (Stucchi, 2002; Stucchi et al., 2003) y las Formaciones Chilcatay del Mioceno Medio y Otuma del Eoceno Tardío-Oligoceno Temprano de Perú (Acosta Hospitaleche y Stucchi, 2005).

Específicamente, en el caso de Chile, la mayor parte de los registros provienen de la misma unidad que los restos aquí estudiados, la Formación Bahía Inglesa, ubicada en la zona costera de la III Región de Atacama (Fig. 1; Rojo, 1985; Marquardt et al., 2000) cuya edad fue recientemente ampliada al Mioceno Medio-Plioceno a partir de un estudio integral de las facies sedimentarias, los cambios del nivel del mar y el análisis geoquímico de nódulos de manganeso (Achurra, 2004). Los restos conocidos previamente al presente hallazgo en la Formación Bahía Inglesa han sido asignados a 1 . Spheniscidae cf. Spheniscus Brisson (1760), 2. Pygoscelis Wagler (1832), 3. Palaeospheniscus Moreno y Mercerat (1891), 4. Paraptenodytes robustus Ameghino (1905), 5. Paraptenodytes antarctica Moreno y Mercerat (1891), 6. Spheniscus megaramphus Stucchi et al. (2003), 7. Spheniscus urbinai Stucchi (2002) y 8. Spheniscus sp. Brisson (1760) (Walsh y Hume, 2001; Acosta Hospitaleche et al., 2002; Acosta Hospitaleche y Tambussi, 2004; Walsh, 2004; Acosta Hospitaleche y Canto, 2005; Chávez, 2005a).

Una cantidad mucho menor de restos provienen de dos afloramientos de la Formación Coquimbo del Mioceno Medio-Plioceno Tardío en la III y IV regiones (Tambussi et al., 2005; Chávez, 
2005b) y del Plioceno Tardío de la Península de Mejillones, originalmente asignados a la Formación Caleta la Herradura (Krebs et al., 1992), de donde fueron exhumados los restos de Spheniscus chilensis Emslie y Guerra Correa, 2003.

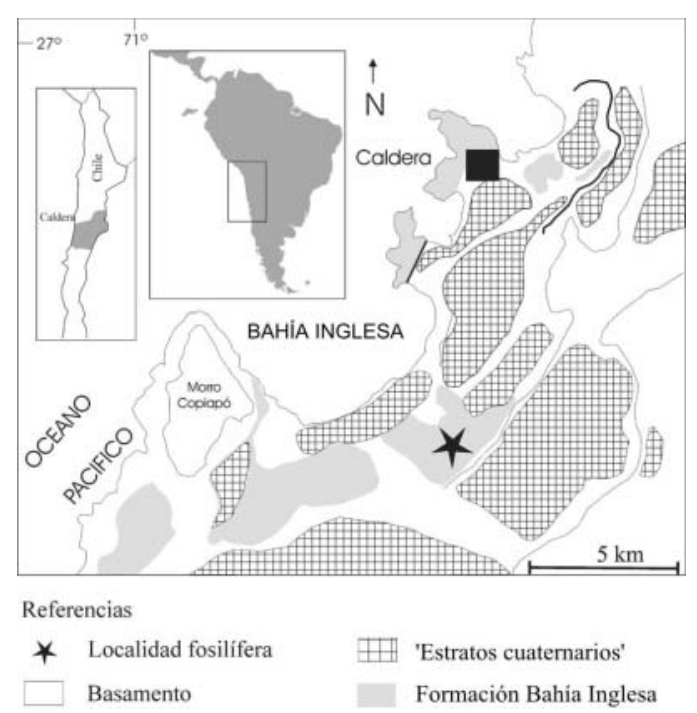

FIG. 1. Mapa de ubicación del área donde fueron colectados los materiales asignados a Pygoscelis calderensis sp. nov. (modificado de Suárez et al., 2004)

Una de las problemáticas centrales en el estudio de los Spheniscidae surge a partir de las características del registro paleontológico. La mayoría de los pingüinos fósiles se conocen a partir de elementos aislados del esqueleto apendicular, motivo por el cual las diagnosis de la mayoría de las especies extintas se basan exclusivamente en los caracteres presentes en los húmeros y/o en los tarsometatarsos (ver Simpson, 1946, 1972a), precisamente los elementos que más fácilmente se preservan en los sedimentos.

Muy escasos son los registros de partes articuladas y de restos craneales. En los casos de Paraptenodytes antarctica (Moreno y Mercerat, 1891) y Marplesornis novaezealandiae (Marples, 1960) se han descrito elementos craneales de morfología claramente disímil con las formas actuales. Los cráneos fósiles más completos corresponden a Spheniscus urbinai y S. megaramphus, ambos procedentes de la Formación Pisco de Perú del Mioceno Tardío (Stucchi, 2002; Stucchi et al., 2003). Se han reportado también otros elementos craneales procedentes de la Formación Bahía Inglesa, afines con el género Spheniscus (Walsh y Hume, 2001), además de siete cráneos presumiblemente pertenecientes a Palaeospheniscus (Acosta Hospitaleche y Canto, 2005) y de un esqueleto completo totalmente articulado asignado a un nuevo taxón proveniente de la Patagonia Argentina, que incluye un cráneo prácticamente completo (Cozzuol et al., 1993).

Por este motivo, los hallazgos de elementos craneanos como los aquí estudiados constituyen importantes contribuciones al avance del conocimiento de la anatomía y la diversidad de la fauna de pingüinos fósiles.

La presente contribución describe tres nuevos restos craneanos exhumados de la Formación Bahía Inglesa. Estos materiales se encuentran depositados en las colecciones de vertebrados de la Sección de Paleontología (SGO-PV) del Museo Nacional de Historia Natural de Santiago de Chile, bajo los números SGO-PV 790, SGO-PV 791 y SGO-PV 792.

Se adopta el criterio sistemático de Simpson (1946, 1972a) y Acosta Hospitaleche (2004) para las especies fósiles, y el de Martínez (1992) para los taxones actuales. La terminología osteológica empleada es la propuesta por Baumel y Witmer (1993) y las medidas, expresadas en milímetros, fueron tomadas con un calibre Vernier de $0,01 \mathrm{~mm}$ de incremento.

Nuestros estudios permitieron la asignación de los materiales a una nueva especie del género actual Pygoscelis Wagler, 1832, que existiría desde el Plioceno Tardío de Nueva Zelanda de confirmarse la antigüedad de los restos asignados a Pygoscelis tyreei (Simpson, 1972b; véase también McKee, 1987). En la actualidad, este género se encuentra representado por tres especies, Pygoscelis antarctica (Forster, 1781), P. papua (Forster, 1781) y $P$. adeliae (Hombron y Jacquinot, 1841), habitantes de aguas antárticas y subantárticas. Presentan preferencias ecológicas similares, en cuanto a la conformación de colonias reproductivas y sus patrones alimentarios basados en el consumo de plancton (Williams, 1995). Los materiales de comparación empleados en el presente estudio se encuentran depositados en las colecciones indicadas en la tabla 1. 
TABLA 1. MATERIALES DE COMPARACIÓN DE LOS CRÁNEOS DE LAS DISTINTAS ESPECIES DE SPHENISCIDAE $\left({ }^{*}\right)$.

\begin{tabular}{|c|c|}
\hline Especie & Materiales \\
\hline Spheniscus magellanicus & $\begin{array}{l}\text { IZUA 0395, MLP A2, MLP A3, MLP A31, MLP A34, MLP A35, IAA5, IAA6, IAA7, } \\
\text { IAA23, MACN 52767, MACN 54681, MACN 54682, MACN 54683, MACN 54685, } \\
\text { MEF 0063. }\end{array}$ \\
\hline Spheniscus humboldti & IZUA 0591, MLP 686 \\
\hline Pygoscelis adeliae & $\begin{array}{l}\text { MLP A415, MLP A416, MLP A417, MLP A418, MLP A419, MLP A420, MLP A421, } \\
\text { MLP A422, MLP A423, MLP A424, MLP A425, MLP A426, MLP A427, MLP A428, } \\
\text { MLP A429, MLP A430, MLP A431, MLP A432, MLP A433, MLP A434, IAA18, IAA21 }\end{array}$ \\
\hline Pygoscelis papua & MLP A449, MLP A450, MLP A451, MLP A463 \\
\hline Pygoscelis antarctica & $\begin{array}{l}\text { IZUA 0343, IZUA 0592, MLP A435, MLP A436, MLP A437, MLP A438, MLP A439, } \\
\text { MLP A440, MLP 441, MLP A442, MLP A443, MLP A444, MLP A445, MLP A446, } \\
\text { MLP A447, MLP A448, IAA8, IAA19, IAA20, IAA22 }\end{array}$ \\
\hline Eudyptes crestatus & MLP A39, IAA 1, IAA 2, IAA 3, IAA 4, MACN 53556 \\
\hline Aptenodytes patagonicus & IAA 9 \\
\hline Aptenodytes forsteri & IZUA 044, IZUA 045, IZUA 046, IZUA 047 \\
\hline Paraptenodytes antarctica & calcotipo MEF- PV del AMHN 3338 \\
\hline Spheniscidae cf. Palaeospheniscus & $\begin{array}{l}\text { SGO-PV 1013, SGO- PV 1022, SGO-PV 1035, SGO-PV 1036, SGO-PV 1051, SGO- } \\
\text { PV } 1053\end{array}$ \\
\hline Spheniscidae sp. nov. & MEF- PV 100 \\
\hline
\end{tabular}

(*) División Paleontología Vertebrados del Museo de La Plata (MLP) de la Ciudad de La Plata, Argentina; Departamento de Ornitología del Museo de Ciencias Naturales 'Bernardino Rivadavia' (MACN) de la Ciudad Autónoma de Buenos Aires, Argentina; Museo Paleontológico ‘Egidio Feruglio' (MEF) de Trelew, Argentina; Instituto Antártico Argentino (IAA) de la Ciudad Autónoma de Buenos Aires, Argentina; Instituto de Zoología de la Universidad Austral, Valdivia, Chile (IZUA) y Sección Paleontología del Museo Nacional de Historia Natural de Santiago, Chile (MNHN).

\section{PROCEDENCIA ESTRATIGRÁFICA}

Los restos craneales aquí estudiados provienen de la Formación Bahía Inglesa (Fig.1; Rojo, 1985; enmendada por Marquardt et al., 2000). Esta unidad contiene depósitos de coquinas, arenas, intercaladas con diatomitas, fosforitas y, ocasionalmente, cenizas retrabajadas (Fig. 2). Esta formación es altamente fosilífera, presentando niveles con abundante fauna marina tanto de vertebrados (Walsh y Hume, 2001; Suárez et al., 2004; Acosta Hospitaleche y Canto, 2005) como de invertebrados (Herm, 1969; Guzmán et al., 1999, 2000; Marchant et al., 2000).
Entre los vertebrados se conocen teleósteos, seláceos, reptiles, cetáceos y pinnípedos (Quilodrán y Marquardt, 2001; Suárez et al., 2004), mientras que la avifauna está representada por restos asignados a los siguientes taxones: 1 . Sula Brisson, 1760; 2. Phalacrocorax Brisson, 1760; 3. Diomedea Linnaeus, 1758 (Walsh y Hume, 2001); 4. Morus Vieillot, 1816 (Walsh, com. pers., 2001); 5. Pseudodontornis cf. longirostris Spulski, 1910 (Chávez y Stucchi, 2002); 6. Diomedeidae indet. aff. Thallasarche; 7. Pelagornis Lartet 1857; 8. Puffinini sensu Penhallurick y Wink, 2004 gén. 


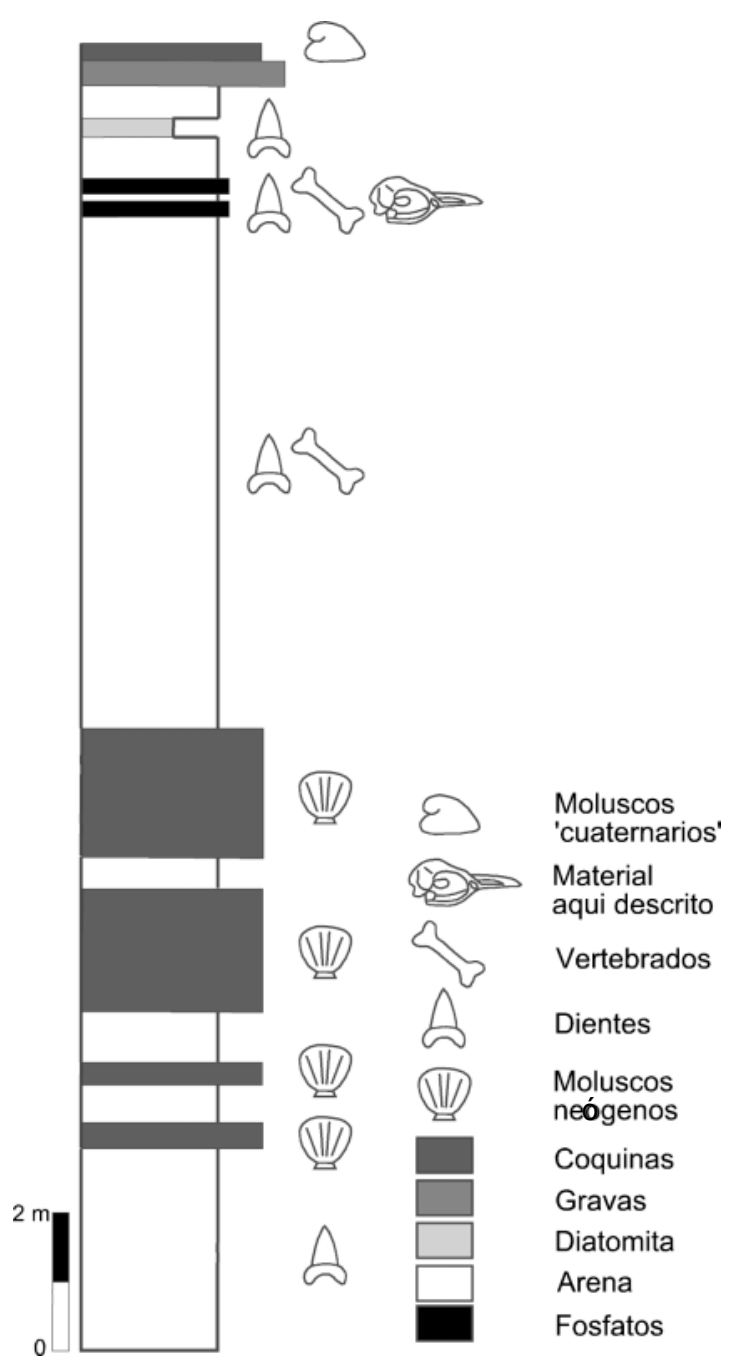

FIG. 2. Columna estratigráfica generalizada de la localidad bajo estudio.

et sp. indet. (Chávez, 2005a) y 9. varias especies de Spheniscidae (Acosta Hospitaleche et al., 2002; Acosta Hospitaleche y Tambussi, 2004; Suárez et al., 2004; Chávez 2005a; Acosta Hospitaleche y Canto, 2005).

Los presentes materiales proceden de los niveles fosfáticos de la formación, en la localidad de
Los Dedos, al oeste de las inmediaciones de la Mina La Fosforita $\left(27^{\circ} 00^{\prime} \mathrm{S}, 70^{\circ} 45^{\prime} \mathrm{W}\right.$ a $28^{\circ} 00^{\prime} \mathrm{S}$, $\left.71^{\circ} 00^{\prime} \mathrm{W}\right)$. El área se caracteriza por la alta concentración de huesos fósiles particularmente en los niveles fosfáticos, que yacen en superficie, ocasionalmente a menos de un metro de la actual superficie de erosión de las terrazas. Restos de vertebrados (teleósteos, seláceos, pinnípedos, cetáceos, reptiles y aves) probablemente procedentes de los mismos niveles que Pygoscelis calderensis han sido ya reportados para esta localidad (Walsh y Hume, 2001; Suárez et al., 2004). Son también comunes las trazas fósiles como los Skolithos en las arenas que se encuentran por encima y por debajo de estas capas portadoras de restos óseos (Walsh y Hume, 2001; Achurra, 2004).

La Formación Bahía Inglesa cubre discordantemente a las 'Gravas de Angostura' (Mioceno Inferior-Medio), $15-20 \mathrm{~km}$ al sur de Caldera, y subyace discordantemente a los estratos cuaternarios de Caldera (Marquardt et al., 2000; Godoy et al., 2003).

La presencia de Globigerina bulloides d'Orbigny, 1826 y Neogloboquadrina pachyderma (Ehrenberg, 1894) permite asignar la base de la secuencia al Mioceno Medio; mientras que Globigerinella calida (Parker, 1962) y Globorotalia crassaformis (Galloway y Wissler, 1927), indican que el techo de la secuencia corresponde al Plioceno Inferior (Marchant et al., 2000; ver también Achurra, 2004).

De acuerdo con los datos micropaleontológicos, los depósitos de esta formación representarían ambientes marinos sublitorales a neríticos con rangos batimétricos entre los 50-500 m (Marchant et al., 2000). Concordantemente con lo estimado a partir de la fauna de invertebrados, los seláceos registrados sugieren un ambiente marino litoral, con profundidades menores a los $200 \mathrm{~m}$ (Walsh y Hume, 2001). La microfauna señala además, para estos niveles, la existencia de períodos con condiciones de aguas subantárticas y otras cálidas (Guzmán et al., 1999; Marchant et al., 2000; Suárez et al., 2004). 


\title{
DESCRIPCIONES SISTEMÁTICAS
}

\author{
Clase Aves Linnaeus, 1758 \\ Orden Sphenisciformes Sharpe, 1891 \\ Familia Spheniscidae Bonaparte, 1856 \\ Género Pygoscelis Wagler, 1832
}

Especie tipo: Pygoscelis papua (Forster, 1781). Holoceno; ca. 6.000 años; Montalti et al., 2004 (islas, Shetland del Sur, Península Antártica) Actual (zona circumpolar; 46-655; Williams, 1995).

\section{Pygoscelis calderensis sp. nov.}

FIG. 3

Holotipo: SGO-PV 790, cráneo carente de la región rostral (recolectado por uno de los autores, $M C)$.

Materiales asignados: SGO-PV 791, cráneo incompleto (citado en publicaciones previas como SPACH FSP-51; Fritis, 2001; Acosta Hospitaleche et al., 2002) y SGO-PV 792, fragmento de basicráneo (recolectados y cedidos al MNHN por los aficionados Fernando Suárez y Ramón Hurtado, respectivamente).

Localidad geográfica: niveles fosfáticos del sector 'Los Dedos', correspondiente a la serie de terrazas sedimentarias interrumpidas por quebradas de profundidad variable, ubicadas al oeste de las inmediaciones de la mina La Fosforita, Bahía Inglesa, Región de Atacama, Provincia de Copiapó, (Fig. $1,27^{\circ} 00^{\prime} \mathrm{S}, 70^{\circ} 45^{\prime} \mathrm{W}$ a $28^{\circ} 00^{\prime} \mathrm{S}, 7^{\circ} 00^{\prime} \mathrm{W}$ ).

Procedencia estratigráfica y edad: Formación Bahía Inglesa (Rojo, 1985; Fig. 1. Mioceno MedioPlioceno (Marchant et al., 2000; Achurra, 2004) .

Diagnosis: fossa glandulae nasalis profunda y limitada por un reborde supraorbitario de ancho constante de unos $20 \mathrm{~mm}$ aproximadamente y más delgado que en las especies actuales del género, en las cuales es mayor que $34 \mathrm{~mm}$ (Tabla 2). Las frontales forman una varilla de alrededor de $1 \mathrm{~mm}$ en la región interorbitaria, más delgada que en $P$. antarctica y $P$. papua, en las cuales alcanza los 3 $\mathrm{mm}$. Fossa temporalis superficial y angosta, de aproximadamente $10 \mathrm{~mm}$, en relación a las demás especies del género, en las que alcanza más de 15 $\mathrm{mm}$. Processus postorbitalis más angostos que en $P$. papua y $P$. antarctica (en los cuales son más triangulares), dirigidos ventralmente y divergentes en su región distal respecto al plano sagital, como en $P$. papua, mientras que en $P$. adeliae y $P$. antarctica son paralelos al plano sagital. Processus paroccipitalis proyectados más ventralmente y prominentia cerebelaris redondeada, a diferencia de las demás especies del género, en las que es aguzada.

Etimología: nombrada en alusión a la ciudad de Caldera, Provincia de Copiapó, Región de Atacama, Chile; próxima a la localidad fosilífera donde se exhumó el holotipo.

Descripción comparada: la comparación con otros pingüinos fósiles, descarta la afinidad del presente taxón con los 'paraptenodytinos' y los 'palaeeudytinos'. Paraptenodytes antarctica Moreno y Mercerat, 1891 presenta las fossae glandulae nasalis derecha e izquierda más ampliamente separadas entre sí (ca. $10 \mathrm{~mm}$ ), es decir, sin acercarse a la línea sagital en el techo craneal (Simpson, 1946), junto con una prominentia cerebelaris mucho más retraída que en las especies vivientes; distinguiéndolos estos caracteres del nuevo material. Palaeeudytes presenta la región interorbitaria mucho más ancha (Marples, 1960) que la de $P$. calderensis, de más de $10 \mathrm{~mm}$. Por su parte, Spheniscus urbinaiy S. megaramphus presentan los frontales más expandidos a nivel interorbitario que $P$. calderensis, como las espe- 
cies actuales del género Spheniscus. Además, al igual que el nuevo taxón (MEF-PV 100) procedente de Patagonia que actualmente se encuentra bajo estudio (Cozzuol et al.,1993), Spheniscus carece de reborde supraorbitario en la fossa glandulae nasalis.

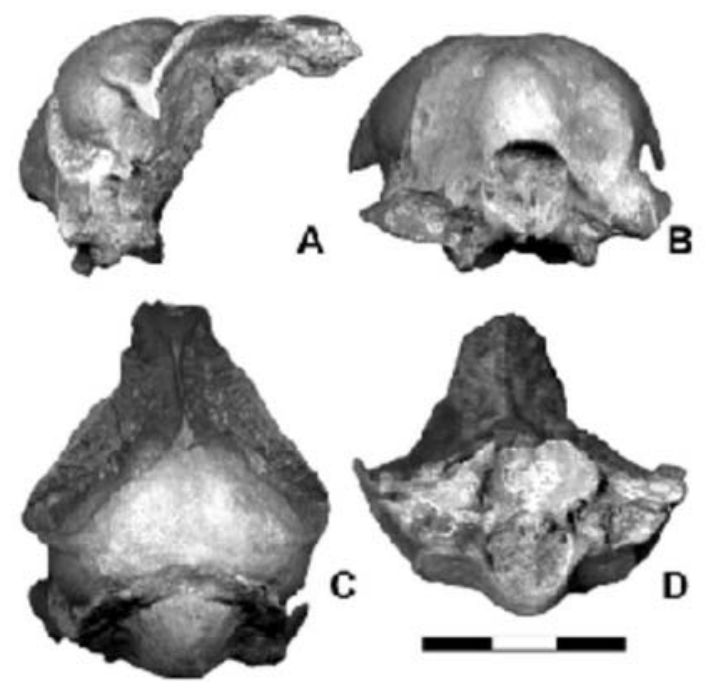

FIG. 3. Cráneo SGO - PV 790 Pygoscelis calderensis sp. nov.: A. vista lateral; B. vista caudal; C. vista dorsal; D. vista ventral. Escala: $30 \mathrm{~mm}$.

La morfología general del cráneo en lo que respecta a la configuración de la fossa glandulae nasalis, el reborde supraorbitario, la disposición y grado de desarrollo de la crista nuchalis temporalis concuerdan con aquella que presentan los géneros actuales antárticos y circumpolares de tamaño pequeño (Pygoscelis y Eudyptes). Contrariamente, en los géneros habitantes de aguas templadas (Spheniscus y Eudyptula), el margen posterior de la fossa glandulae nasalis es proporcionalmente más angosto, llegando a desaparecer hacia su porción más posterior, y los frontales se encuentran mucho más expandidos a la altura de las órbitas que en Pygoscelis sp.

Además, la fossa glandulae nasalis presenta una extensión anteroposterior mayor que en Eudyptes Vieillot, 1816, en el cual aparece delimitada posteriormente, mientras que en el nuevo material se encuentra abierta. La presencia de un reborde supraorbitario permite también diferenciarlo de los géneros vivientes Spheniscus, Eudyptula Bonaparte, 1856, Aptenodytes Miller, 1778 y Megadyptes Milne-Edwards, 1880.

La morfología y el grado de desarrollo de las fossae glandulae nasalis, la disposición relativa de las cristae nuchalis temporalis y la configuración de la región occipital permiten asignar los nuevos materiales al género Pygoscelis Wagler, 1832.

A nivel específico, las fossae glandulae nasalis de $P$. calderensis poseen rebordes supraorbitarios más delgados que en las especies actuales del género, ca. $2 \mathrm{~mm}$. Asimismo, las fosas derecha e izquierda se encuentran separadas entre sí por medio de un frontal muy delgado (de menos de 1 $\mathrm{mm}$ ) en forma de varilla, como en $P$. adeliae, mientras que en $P$. antarctica y $P$. papua se desarrollan frontales más anchos (de hasta $4 \mathrm{~mm}$ ) en forma de placa. El extremo posterior de esta fosa posee forma redondeada como en $P$. papua y $P$. adeliae y no aguzada como en $P$. antarctica. Los processus postorbitalis de $P$. calderensis se dirigen ventralmente y divergen de la línea sagital en sus extremos distales como en $P$. papua, mientras que en $P$. adeliae y $P$. antarctica se proyectan ventrocaudalmente. Estos procesos son triangulares y anchos ( ca. 5,5 mm) en $P$. adelia y en $P$. papua, pero delgados (ca. $4,4 \mathrm{~mm}$ ) en $P$. antarctica y en $P$. calderensis. Los processus paroccipitalis se encuentran proyectados ventralmente en el nuevo material, a diferencia de $P$. papua, donde se dirigen hacia la región craneal; 0 en $P$. antarctica y $P$. adeliae donde se dirigen hacia la región caudal. La prominentia cerebelaris es más redondeada caudalmente que en $P$. papua y no aguzada como en $P$. adeliae. La fossae temporalis de $P$. calderensis no se encuentra delimitada anteriormente por una cristae nuchalis temporalis como en $P$. antarctica, sino que permanecen abiertas como en $P$. papua. Las cristae nuchalis temporalis y la cristae nuchalis transversa enmarcan una fossa temporalis más amplia en sentido craneocaudal en $P$. calderensis que en cualquier otra especie de Pygoscelis.

Los únicos restos fósiles descritos para este género corresponden a elementos poscraneales asignados a Pygoscelis tyreei Simpson, 1972a, del Plioceno Temprano de Nueva Zelanda 
(Simpson, 1972b). Si bien no resulta posible realizar una comparación directa con los materiales aquí estudiados, se puede afirmar que Pygoscelis calderensis sp. nov. habría sido un ave de menor talla que las especies actuales del género, mientras que $P$. tyreei habría alcanzado un tamaño similar al de la especie viviente $P$. papua (Simpson, 1972b). Resulta importante señalar que Pygoscelis tyreei proviene de sedimentos que Simpson (1972b) habría asignado al Plioceno, pero que actualmente se estima que podrían corresponder a un lapso temporal variable entre el Mioceno Medio y el Pleistoceno Temprano, ya que la geología de la zona no se encuentra aún estudiada en detalle (McKee, 1987). No sólo resulta dudosa la antigüedad de $P$. tyreei, sino que su asignación sistemática al género Pygoscelis no puede ser tampoco considerada como certera hasta tanto no se cuente con materiales diagnósticos para su reestudio (Simpson, 1972b). Esta duda resulta razonable dado que por un lado, el propio Simpson (1972b) consideró tan solo como preliminar su asignación a $P$. tyreii, y por el otro, a que los materiales sobre los cuales fue fundada esa especie no incluyen tarsometatarsos ni cráneo y sólo un húmero fragmentado entre todos los elementos que puedan considerarse como diagnósticos.

Por otra parte, recientemente han sido dados a conocer restos poscraneales procedentes de la Formación Bahía Inglesa asignados a Pygoscelis sp. que aunque se encuentran aún bajo estudio, corresponderían a un individuo adulto cuya talla sería comparable a la de Aptenodytes patagonicus (Miller, 1778) y por tanto, claramente distinguible de la de Pygoscelis calderensis (Walsh, 2004). Medidas: en la tabla 2 se listan las dimensiones de los materiales asignados a $P$. calderensis. Medidas homólogas fueron tomadas en las especies actuales del género a modo comparativo.

TABLA 2. MEDIDAS (EN MILÍMETROS) DE LOS EJEMPLARES ASIGNADOS A PYGOSCELIS CALDERENSIS SP. NOV.

\begin{tabular}{|lcccccc|}
\hline \multicolumn{1}{|c}{ Medidas } & $\begin{array}{c}\text { SGO- } \\
\text { PV 790 }\end{array}$ & SGO-PV 791 & SGO-PV 792 & $\begin{array}{c}\text { Pygoscelis } \\
\text { adeliae }\end{array}$ & P. papua & P. antarctica \\
& & 52,2 & & 47,8 & 51,1 & 44,4 \\
\hline Longitud frontales & 50,3 & & & 44,1 & 46,5 & 43,6 \\
Ancho frontales & 17,5 & 22,7 & & 22,3 & 20,4 & 19,2 \\
Altura supraoccipital & 16 & 17 & 15,7 & 35,0 & 35,1 & 34,7 \\
Ancho supraoccipital & 12,8 & 14,9 & & 12,4 & 11,8 & 10,3 \\
Ancho fossa glandulae nasalis & 12,8 & 19,3 & 20,5 & 18,5 & 15,8 \\
Ancho lámina parasphenoidalis & 20,8 & 21 & & 0,62 & 0,34 & 0,41 \\
Ancho reborde supraorbitario & 0,19 & 0,21 & 41 & 53,9 & 54,8 & 51,5 \\
Ancho postorbitario & 47,2 & 56 & 49 & 36,1 & 36,4 & 33,7 \\
Altura & 31,6 & 44 & 39 & & & \\
\hline
\end{tabular}

\section{DISCUSIÓN Y CONCLUSIONES}

La comparación con los taxones fósiles y vivientes, permite la asignación de los presentes materiales al género actual Pygoscelis debido a la presencia de una fossa glandulae nasalis ancha y limitada externamente por un reborde supra orbitario y de fosas temporales de menos de 4,5 $\mathrm{mm}$ en su porción posterior, delimitadas por las cristae nuchalis temporalis que no alcanzan la línea sagital (ver caracteres métricos en tabla 2). Asimismo, el desarrollo de profundas fossae glandulae nasalis, el ancho constante del reborde supraorbitario, el desarrollo de angostos frontales, la disposición ventral y divergente de los delgados processus postorbitalis, la proyección ventral de los processus paroccipitalis y la forma redondeada de la prominentia cerebelaris, permiten proponer una nueva especie.

Pygoscelis calderensis se distingue claramente de los especímenes presentados por Walsh (2004) como Pygoscelis sp. nov. por una marcada 
diferencia de tamaños. $P$. calderensis habría sido ligeramente menor que las especies actuales del género, mientras que la especie fósil del Plioceno Tardío de Nueva Zelanda, P. tyreei, habría sido similar a la especie actual $P$. papua en su tamaño y morfología general (Simpson, 1972b).

Actualmente, el género Pygoscelis posee una distribución meridional restringida a áreas antárticas y subantárticas, siendo $P$. papua, la especie cuya distribución se extiende más hacia el norte, alcanzando la costa atlántica de América del Sur $\left(43^{\circ} \mathrm{S}\right)$ y las islas al sur de Nueva Zelanda. Las principales colonias reproductivas de $P$. papua se encuentran en Antártica; sin embargo, también existen colonias en el archipiélago de las Malvinas, isla Georgia del Sur e isla Martillo en el Canal Beagle (Couve y Vidal, 2003). Las restantes especies vivientes, $P$. antarctica y $P$. adeliae, se encuentran restringidas al continente antártico, siendo accidentales su presencia en el territorio subantártico.

El registro fósil sugiere claramente que el género se encontraba más ampliamente distribuido durante el Neógeno, siendo evidencia de ello la

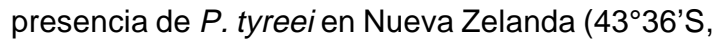
$\left.173^{\circ} 36^{\prime} \mathrm{E}\right)$ y de $P$. calderensis en el norte de Chile $\left(27^{\circ} 00^{\prime} \mathrm{S}, 70^{\circ} 45^{\prime} \mathrm{W}\right.$ a $\left.28^{\circ} 00^{\prime}, 71^{\circ} 00^{\prime} \mathrm{W}\right)$. De esta manera, puede afirmarse que el presente hallazgo amplía la distribución latitudinal del género hasta los $27^{\circ} \mathrm{S}$ en el Pacífico sureste. Un caso similar ocurre con el género Aptenodytes, de actual distribución antártica y subantártica, para el cual se conoce (A. ridgeni Simpson 1975) un representante fósil del Plioceno de Nueva Zelanda. La comparación entre estos dos géneros resulta oportuna, dada su actual distribución parcialmente simpátrica y su proximidad filogenética (Acosta Hospitaleche, 2004; ver también Giannini y Bertelli, 2004; Bertelli y Giannini, 2005).

Es posible que el retroceso en la distribución geográfica de estos géneros típicamente antárticos se haya producido paulatinamente a lo largo de la fase final del Neógeno. Sin embargo, el conocimiento es aún insuficiente para determinar el momento actual exacto en que esto habría ocurrido. Por otra parte, los aportes efectuados a partir de la avifauna cuaternaria de pingüinos de Nueva Zelanda y Antártica, señalan la presencia de las especies actuales del género Pygoscelis en sitios comprendidos dentro de su distribución actual que se extiende hacia el norte hasta los $43^{\circ}$ (Fordyce, 1991; Acosta Hospitaleche et al., 2004).

Dada la estrecha asociación de la totalidad de las especies modernas del género (Pygocelis adeliae, $P$. antarctica y $P$. papua) con los ambientes y corrientes de aguas frías, se estima que $P$. calderensis habría habitado las costas de Chile en momentos en los que las corrientes de agua fría ya se habían instaurado en el área.

La abundancia de restos de Spheniscidae en estos depósitos, que seguramente habrían constituido extensas colonias en el área, así como la presencia de diversos Procellariformes (Chávez, 2005a), son también indicadores de ambientes fríos o, al menos, del establecimiento de corrientes de aguas frías en el área, como sucede actualmente. Esta hipótesis concuerda con lo sugerido previamente a partir de los estudios micropaleontológicos (Marchant et al., 2000; Tsuchi, 2002).

Aunque los ítems alimentarios de Pygoscelis varían de una localidad a otra, su dieta se encuentra dominada esencialmente por organismos planctónicos. La presencia de Pygoscelis calderensis en el área de estudio, permite pensar en la disponibilidad de este recurso en los mares durante el Neógeno. Adicionalmente, la gran cantidad de restos de Mysticetos y la presencia de otros vertebrados que se alimentan de plancton, tales como los petreles paloma Pachyptila y los tiburones peregrinos Cetorhinus sp. sustentan esta idea (Suárez y Marquardt, 2003).

Finalmente, es importante mencionar, que a partir del hallazgo de Pygoscelis calderensis sp. nov., los géneros Pygoscelis y Spheniscus se convierten en los únicos taxones modernos que se registran en el Mioceno Medio. Estos registros, son los más antiguos para cualquier género actual y corresponden, ambos, a localidades sudamericanas emplazadas sobre la costa pacífica. 


\section{AGRADECIMIENTOS}

A D. Frassinetti (Museo Nacional de Historia Natural de Santiago, Chile) por el acceso a las colecciones de la Formación Bahía Inglesa. A R. Schlatter y R. Ulbrich (Universidad Austral de Chile) por el acceso a los materiales de comparación.
A los árbitros $\mathrm{H}$. Alvarenga (Universidad de Sao Paulo, Brasil), C. Marquardt (Servicio Nacional de Geología y Minería de Santiago, Chile) y C. Tambussi (Museo de La Plata, Argentina).

\section{REFERENCIAS}

Achurra Reveco, L. 2004. Cambios del nivel del mar y evolución tectónica de la cuenca neógena de Caldera, III Región. Tesis de Magíster (Inédito), Universidad de Chile, Departamento de Geología: 138 p.

AcostaHospitaleche, C. 2003. Paraptenodytes antarcticus (Aves, Sphenisciformes) en la Formación Puerto Madryn (Mioceno tardío temprano), provincia de Chubut, Argentina. Revista Española de Paleontología 18: 179-183.

Acosta Hospitaleche, C. 2004. Los pingüinos (Aves, Sphenisciformes) fósiles de Patagonia. Sistemática, biogeografía y evolución. Tesis Doctoral (Inédito), Universidad Nacional de La Plata, Facultad de Ciencias Naturales y Museo: 321 p. La Plata.

Acosta Hospitaleche, C. 2005. Systematic revision of Arthrodytes Ameghino, 1905 (Aves, Spheniscidae) and its assignment to the Paraptenodytinae. Neues Jahrbuch für Geologie und Paläontologie 7: 404- 414.

Acosta Hospitaleche, C.; Canto, J. 2005. Primer registro de cráneos de Palaeospheniscus (Aves, Spheniscidae), procedentes de la Formación Bahía Inglesa (Mioceno medio-tardío). Revista Chilena de Historia Natural 78: 489-495. Santiago.

Acosta Hospitaleche, C.; Fritis, O.; Tambussi, C.; Quinzio, A. 2002. Nuevos restos de pingüinos (Aves, Spheniscidae) en la Formación Bahía Inglesa (Mioceno superior-Plioceno inferior) de Chile. In Congreso Latinoamericano de Paleontología de Vertebrados, No. 1, Resúmenes: 16. Santiago.

Acosta Hospitaleche, C.; Stucchi, M. 2005. Nuevos restos terciarios de Spheniscidae (Aves, Sphenisciformes) procedentes de la costa del Perú. Revista Española de Paleontología 20: 1-5.

Acosta Hospitaleche, C.; Tambussi, C. 2004. Fossil Penguins from South America. In International Penguin Conference, No. 5, Abstracts Book: 48. Tierra del Fuego.

Acosta Hospitaleche, C.; Tambussi, C.; Cozzuol, M. 2004. Eretiscus tonnii Simpson 1981 (Aves, Sphenisciformes): materiales adicionales, status taxonómico y distribución geográfica. Revista del
Museo Argentino de Ciencias Naturales 6: 632-637. Ameghino, F. 1905. Enumeración de los impennes fósiles de Patagonia y de la isla Seymour. Anales del Museo Nacional de Buenos Aires 6: 97-167.

Baumel, J.; Witmer, L. 1993. Osteologia. In Handbook of avian anatomy: Nómina Anatómica Avium (Baumel, J.; King, A.; Breazile, J.; Evans, H.; Vanden Bergue, J.C.; editors). Nuthall Ornithological Club: 45-132. Cambridge, Massachusetts.

Bertelli, S.; Giannini, N. 2005. A phylogeny of extant penguins (Aves, Sphenisciformes) combining morphology and mitochondrial sequences. Cladistics 21: 209-239.

Bonaparte, C.L. 1856. Espéces nouvelles d'Oiseaux d'Asie et d'Amérique et tableaux para lléliques des Pélagiens ou Gavae. Comptes Rendus hebdomadaires des Séances de l'Académie des Sciences, 42: 764-776.

Brisson, M. 1760. Ornithologie; ou, Methode contenant la division des oiseaux en ordres, sections, genres, especes et leurs varietes 1: $632 \mathrm{p}$.

Chávez, M. 2005a. Nuevos registros de aves fósiles en la Formación Bahía Inglesa (Mioceno-Plioceno), Región de Atacama, Chile. In Congreso Chileno de Ornitología, No. 8, Actas: 47. Chillán.

Chávez, M. 2005b. Una nueva localidad con aves fósiles en la Región de Atacama, Chile. In Congreso Chileno de Ornitología, No. 8, Actas: 47. Chillán.

Chávez, M.; Stucchi, M. 2002. El registro de Pelagornithidae (Aves, Pelecaniformes) en el Pacífico Sudeste. In Congreso Latinoamericano de Paleontología de Vertebrados, No. 1, Resúmenes: 28. Santiago.

Cione, A. L.; Tonni, E. P. 1981. Un pingüino de la Formación Puerto Madryn (Mioceno tardío) de Chubut, Argentina. Comentarios acerca del origen, la paleoecología y zoogeografía de los Spheniscidae. In Anales Congreso Latinoamericano de Paleontología, Actas 2: 591-604. Porto Alegre.

Couve, E.; Vidal, C. 2003. Aves de Patagonia, Tierra del Fuego y Península Antártica. Editorial Fantástico Sur 
Birding Ltda.: $307 \mathrm{p}$.

Cozzuol, M.A.; Tambussi, C.; Noriega, J. 1993. Un pingüino (Aves, Spheniscidae) de la Formación Puerto Madryn (Mioceno Medio) en Península Valdés, Chubut, Argentina, con importantes implicancias filogenéticas. Ameghiniana 30: 327-328.

d'Orbigny, A. D. 1826. Tableau méthodique de la classe des Céphalopodes. Annales des Sciences Naturelles 7: 245-314.

Emslie, S. D.; Guerra Correa, C. 2003. A new species of penguin (Spheniscidae: Spheniscus) and other birds from the late Pliocene of Chile. Proceedings of the Biological Society of Washington 116: 308-316.

Ehrenberg, C.G. 1894. Elemente des tiefen Meeresgrundes in Mexikanischen Golfstrome bei Florida; Ueber die Tiefgrund-Verhältnisse des Oceans am Eingang der Davisstrasse und bei Island. Kaiserlichen Preuss Akademie der Wissenschaften Berlin, Monatsberichte: 222-240.

Fritis, O. 2001. Descripción y análisis de la ornitofauna fósil de la Formación Bahía Inglesa (Mioceno superior), III Región de Atacama, Chile. Tesis de Grado (Inédito), Universidad de Concepción, Facultad de Ciencias Naturales y Oceanográficas: $43 \mathrm{p}$.

Fordyce, E. 1991. A new look at the fossil vertebrate record of New Zealand. In Vertebrate Paleontology of Australasia Nickers-Rich, P.; Monaghan, J. M.; Baird, R.F.; Rich, T.H.; editors), Pioneer Design Studio and Monash University: 1191-1316. Melbourne.

Fordyce, E.; Jones, C. 1990. Penguin history and new fossil material from New Zealand. In Penguin Biology (Davis, L. S.; Darby, I. T.; editors). Academic Press Inc.: 419-446. San Diego.

Forster, J. R. 1781. Historia aptenodytae. Generis avium orbiavstraliproprii. Commentationes Societatis Regiae Scientiarum Gottingensis 3: 121-148.

Galloway, J.; Wissler, S. 1927. Pleistocene foraminifera from the Lomita Quarry, Palos Verdes Hills, California. Journal of Paleontology 1: 35-87.

Giannini, N.P.; Bertelli, S. 2004. Phylogeny of extant penguins based on integumentary and breedings characters. The Auk 121: 421-434.

Godoy E.; Marquardt, C.; Blanco, N. 2003. Carta Caldera: Región de Atacama. Servicio Nacional de Geología y Minería, Carta Geológica de Chile, Serie Geología Básica, escala 1:100.000, 76: 36 p. Santiago.

Guzmán, N.; Frassinetti, D.; Marquardt, C. 1999. Moluscos de edad miocena del área de Caldera, Chile. In Congreso de Malacología, No. 4, Actas: 6566. Coquimbo.

Guzmán, N.; Marquardt, C.; Ortlieb, L.; Frassinetti, D. 2000. La malacofauna neógena y cuaternaria del área de Caldera $\left(27^{\circ}-28^{\circ} \mathrm{S}\right)$ : especies y rangos bioestratigráficos. In Congreso Geológico Chileno, No. 9. Actas 1: 476-481. Puerto Varas.

Herm, D. 1969. Marines Pliözan und Pleistözan in Nord und Mittel Chile unter besonderen Berücksichtigung der Entwicklung der Mollusken-Faunen. Zitteliana 2: 1-159. Munchen.

Hombron, J.; Jacquinot, H. 1841. Description de plusieurs oiseaux nouveaux ou peu connus, provenant de l'expédition autour du monde sur les corvettes L'Astrolabe et La Zélée. Annales Des Sciences Naturelles (Zoologie) 2: 312-320.

Krebs, W.; Aleman, A.; Padilla, H.; Rosenfeld, J.; Niemeyer, H. 1992. Age and paleoceanographic significance of the Caleta Herradura diatomite, Península de Mejillones, Antofagasta, Chile. Revista Geológica de Chile 19 (1): 75-81.

Lartet, E. 1857. Note sur un huméris fossile d'Oiseau, attribué a un tres-grand palmipede de la section des Longipennes. Comptes-rendus hebdomadaires des seances de l'Academie des Sciences: 736-741.

Linnaeus, C. 1758. Systema naturae per regna tria naturae, secundum classes, ordines, genera, species, cum characteribus, differentiis, synonymis, locis. Editio Decima, reformata. Laurentii Salvii 1:824 p. Stockholm.

Marchant, M.; Marquardt, C.; Blanco, N.; Godoy, E. 2000. Foraminíferos del área de Caldera $\left(26^{\circ} 45^{\prime}-28^{\circ} \mathrm{S}\right)$ y su utilización como indicadores cronoestratigráficos del Neógeno. In Congreso Geológico Chileno, No. 9, Actas 1: 499-503. Puerto Varas.

Marples, B.J. 1960. A fossil penguin from the Late Tertiary of North Canterbury. Records of the Canterbury Museum 7 (3): 185-195.

Marquardt, C.; Blanco, N.; Godoy, E.; Lavenu, A.; Ortlieb, L.; Marchant, M.; Guzmán, N. 2000. Estratigrafía del Cenozoico Superior en el área de Caldera $\left(26^{\circ} 45^{\prime}\right.$ $\left.28^{\circ} \mathrm{S}\right)$, III Región de Atacama. In Congreso Geológico Chileno, No. 9, Actas: 504-508. Puerto Varas.

Martínez, I. 1992. Order Sphenisciformes. In Handbook of the birds of the world, Ostrich to Ducks (del Hoyo, J.; Elliott, A.; Sargatal, J.; editors). Lynxs editions 1:140-160. Barcelona.

McKee, J. W. 1987. The occurrence of the Pliocene penguin Terengaiornis moisleyi (Sphenis ciformes: Spheniscidae) at Hawera, Taranaki, New Zealand. New Zealand Journal of Zoology 14: 557- 561.

Miller, J.F. 1778. Various subjects of Natural History, where in are delineated birds, Animals and many curious Plants. Icones Animalium et Plantarum: $10 \mathrm{p}$.

Milne-Edwards, A. 1880. Expéditions scientifiques du Travailleur. Annales Des Sciences Naturelle 6: 56 p.

Montalti, D.; Acosta Hospitaleche, C.; del Valle, R.; Ferman, L. 2004. Fossil penguin remains at the South Shetland Island: Taphonomical Inferences. In International Penguin Conference, No. 5, Abstracts Book: 63. Tierra del Fuego.

Moreno, F. P.; Mercerat, A. 1891. Catálogo de los pájaros fósiles de la República Argentina conservados en el Museo de La Plata. Anales del Museo de La Plata (Paleontología Argentina) 1: 7-71. 
Myrcha, A.; Tatur, A.; Del Valle, R. 1990. A new species of fossil penguin from Seymour Island. West Antarctica. Alcheringa 14: 195-205.

Olson, S.L. 1986. A replacement name for the fossil penguin Microdytes Simpson (Aves: Spheniscidae). Journal of Paleontology 60: 785.

Parker, F. L. 1962. Planktonic foraminiferal species in Pacific sediments. Micropaleontology 8: 219-254.

Penhallurick, J.; Wink, M. 2004. Analysis of the taxonomy and nomenclature of the Procellariformes based on complete nucleotidesequences of the mitochondrial cytochrome b gene. Emu 104: 125-147.

Quilodrán, P.; Marquardt, C. 2001. Catastro y condiciones de fosilización de cetáceos neógenos de la Formación Bahía Inglesa, 27ํ-28오, Tercera Región, norte de Chile. Un aporte al conocimiento del Terciario marino: Formación Bahía Inglesa. Ameghiniana 38 (2): 15-16R.

Rojo, M. 1985. Un aporte al conocimiento del Terciario marino: Formación Bahía Inglesa. In Congreso Geológico Chileno, No. 4, Actas 1: 514-533. Antofagasta.

Sharpe, R. B. 1891. A review of recent attempts to classify birds. In Proceedings of the International Ornithological Congress, No. 2: 90. Budapest.

Simpson, G. G. 1946. Fossil penguins. Bulletin of the American Museum of Natural History 87: 1-100.

Simpson, G. G. 1972a. Conspectus of Patagonian Fossil Penguins. American Museum Novitates (2488): 137.

Simpson, G. G. 1972b. Pliocene penguins from North Canterbury, New Zealand. Records of the Canterbury Museum 9: 159-182.

Simpson, G. G. 1975. Notes on variation in penguins and on fossil penguins from the Pliocene of Langebaanweg. Annals of the South African Museum 69: 59-72. Cape Province. South Africa.

Spulski, B. 1910. Odontopteryx longirostris sp. nov. Zeitschrift der Deutschen Geologie Gesellschaft. Monatsberichte 7: 507-521.

Stucchi, M. 2002. Una nueva especie de Spheniscus (Aves, Spheniscidae) de la Formación Pisco, Perú. Boletín Geológico del Perú 94: 17-24.
Stucchi, M.; Urbina, M.; Giraldo, A. 2003. Una nueva especie de Spheniscidae del Mioceno tardío de la Formación Pisco, Perú. Bulletin Institut Français d'Etudes Andine 32 (2): 361-375.

Suárez M. E.; Lamilla, J.; Marquardt, C. 2004. Peces Chimaeriformes (Chondrichthyes, Holocephali) del Neógeno de la Formación Bahía Inglesa (Región de Atacama, Chile). Revista Geológica de Chile 31 (1): 105-117.

Suárez, M. E.; Marquardt, C. 2003. Revisión preliminar de las faunas de peces elasmobranquios del Mesozoico y Cenozoico de Chile y comentarios sobre su valor cronoestratigráfico. In Congreso Geológico Chileno, No. 10, Sección temática 3, CD-ROM. Concepción.

Tambussi, C.; Acosta Hospitaleche, C.; Canto, J. 2005. Paleornitofauna de pingüinos de Chile. In Congreso Latinoamericano de Paleontología de Vertebrados, No. 2: 259-260. Río de Janeiro.

Tambussi, C.; Reguero, M.; Marenssi, S.; Santillana, S. 2002. The earliest known penguin and the evolution of Spheniscid size. In Congreso Latinoamericano de Paleontología de Vertebrados, No. 1, Resúmenes: 53. Santiago.

Tsuchi, R. 2002. Neogene evolution of surface marine climate in the Pacific and notes on related events. Revista Mexicana de Ciencias Geológicas 19: 260270.

Vieillot, L. P. 1816. Analyse d'une nouvelle ornithologuie elementaire. Nouveau dictionnaire d'histoire naturelle. Nouvelle édition. Deterville, 455 p. París.

Wagler, J. G. 1832. Isis, oder Encyclopaedische Zeitung 25. Editing Pygoscelis (section): $281 \mathrm{p}$.

Walsh, S. 2004. New penguin remains from the Neogene of Chile. Internacional Meeting of the Society of Avian Paleontology and Evolution, No. 6, Abstracts Books: 60-61. Francia.

Walsh, S.; Hume, J. 2001. A new neogene marine avian assemblage from north-central Chile. Journal of Vertebrate Paleontology 21 (3): 484-491.

Williams, T.D. 1995. The penguins. Spheniscidae. Birds families of the world. Oxford University Press: 295 p. 